\title{
Experten geben Tipps für den Labor- und Praxisalltag
}

Besucher der IDS können am Stand der VITA Zahnfabrik hautnah erleben, wie sie innovative Neuprodukte und bewährte VITA-Lösungen bei der Herstellung von Zahnersatz unterstützen. Experten aus Praxis und Labor stellen VITA-Neuheiten vor und demonstrieren deren praktische Anwendungsvorteile. In Live-Demos erhalten Besucher viele wertvolle Tipps. VITA IMPLANT SOLUTIONS (IS) zur Herstellung von implantatgetragenem Zahn- ersatz sind bei den VITA CAD/CAM-Restaurationsmaterialien das Topthema. Ihre Erfahrungen mit den neuen Rohlingen geben die Zahnärzte Peter Neumann und Dr. Dirk Ostermann weiter. Weitere Fachleute geben darüber hinaus Empfehlungen zu VITA SUPRINITY, der zirkondioxidverstärkten CAD/CAM-Glaskeramik, sowie zu VITABLOCS, der CAD/CAM-Feldspatkeramik mit über 25-jähriger Erfolgsgeschichte. Mit diesem reichhaltigen Infor- mations- und Demo-Programm lohnt sich der Besuch am VITA-Stand in jedem Fall. Details zu den jeweiligen Demo-Orten entnehmen Sie dem virtuellen Standplan vor Ort.

Halle 10.1/Stand D010

Nach einer Pressemitteilung der VITA Zahnfabrik H. Rauter GmbH \& Co. KG, Bad Säckingen 